\title{
Creative Process within Globally Distributed Teams: Brazil, Sweden, France and India
}

\author{
Rosana S. Reis ${ }^{1, *}$ \\ ${ }^{1}$ ISG International Business School, Department of Management, Paris 75116, France \\ *Correspondence: ISG International Business School, Department of Management, Paris 75116, France. E-mail: \\ rosana.reis@isg.fr
}

Received: July 25, 2014

Accepted: September 5, $2014 \quad$ Online Published: October 7, 2014

doi:10.5430/mos.v2n1p1

URL: http://dx.doi.org/10.5430/mos.v2n1p1

\begin{abstract}
The current complexity of economic, social, technological and business environments in which modern organizations are inserted have determined new forms of working relationships and configurations. This has in turn triggered numerous studies and comparative analyses about the processes enveloped by these new working patterns. The aim of this study is to explore and analyse how the creative process unfolds in globally distributed teams. Using a qualitative approach, we presented a case study conducted within Volvo 3P projects and more specifically the New Product Development team based in Brazil, which works alongside teams distributed in Sweden, France and India. Our empirical evidence shows that whilst individuals are the core element of the creative process in collocated teams; in globally distributed teams the fundamental foundation of the creative process is the organization.
\end{abstract}

Keywords: creative process; globally distributed teams; international management; qualitative study; case study

The aim of this paper is to explain and understand exactly how the creative process occurs in such a new configuration of working conditions, namely among globally distributed teams (GDT). This paper re-asses the theme by exploring and re-visiting the elements that contribute to the creative process in virtual settings, given recent developments in communication technology. GDT can be defined as interdependent work teams of culturally diverse members based in two or more nations that share collective responsibilities (Baba, Gluesing, Ratner, \& Wagner, 2004), and which are brought together by information and telecommunication technologies to accomplish one or more organizational tasks (Piccoli, Powell, \& Ives, 2004). The creative process will be analysed in relation to the particular activities that occur while a person or team is in the act of creating (Nemiro, 2002).

Presupposing creativity to be a social process, we investigated the way in which this phenomenon occurs when the configuration of the team is substantially modified. With business environments no longer confined to geographical borders, distributed teams overcome the limitations of time, space, and the organizational affiliations of traditional collocated teams (Piccoli et al., 2004). Questions will also be raised as to the ways in which this new conception of work affects the creative process. The participants are dealing with quite an atypical situation, socialization takes place amongst individuals who belong to a geographically dispersed workplace, in which interpersonal relationships are mediated by computer, and trust must be developed among those who have never actually met one another or, as it is said, lack the expected "touch" among the team. Participants not only have multiple addresses and locations, but above all different nationalities, and therefore different cultures, attitudes, thoughts, working patterns and languages. How does the creative process unfold in globally distributed teams? Which are the necessary conditions to work together without ever meeting before?

In order to understand this phenomenon, the present research essentially comprises of a case study of teams from the Business Unit Volvo 3P (Volvo Group) situated in Brazil, Sweden, France and India, which are in charge of developing new products. 


\section{Literature Review}

Creativity is understood as the ability to produce work that is both, novel - i.e. original, unexpected; and appropriate i.e. useful, adaptive concerning task constraints (Sternberg \& Lubart, 1999). In broad terms, creativity may occur in virtually any domain of human activity (Plucker \& Renzulli, 1999). The purpose of this research is to investigate how the creative process occurs in GDT responsible for the development of new products. This review has been organised to provide an overview of the subject in question.

\subsection{The Study of Creativity in the Team Level}

Creativity does not occur spontaneously or randomly, but takes place whenever an individual shows a specific combination of knowledge, skills and motivation for creating new ideas (Kurtzberg \& Amabile, 2001). Triggering creative behavior in collocated teams is a process that can be influenced and managed, but which has clearly is not yet fully understood (Kylen \& Shani, 2002). Indeed, most of the previous researchers in this field who have sought to understand the development of the creative process in teams present various inconsistencies and dualities in their work because they fail to distinguish between ideas created in an individual mind and ideas arising from creative synergy. One camp tends to view the creative process within a team as a collective development, where all participants take up a single identity in team unity. Other researchers tracking the evolution of individual ideas to the team level subscribe to the view that although individuals work together in teams, synergy does not suppress the individuality of the participants.

If we apply both these views to GDT, the question arises as to how synergy can be created in teams located distantly apart from each other. According to Cini (1999), the synergy can be created when leadership education is adapted to the online environment. Although the level of analysis is naturally focused on teams, it should be remembered that a team is formed 'of' and 'by' individuals. This fact shows the need to better understand the creativity of the individual within the team. Under the interactionist model of creativity, individual creativity is influenced by one's prior experiences, cognitive abilities or style, personality, intrinsic motivation and knowledge, which together contribute to farm team creativity.

Teams with a shared sense of teamwork and cohesiveness have a common understanding of team processes, participant roles, and team goals (Peelle III, 2006). Consequently, team members' shared understanding and beliefs can therefore subsequently affect team process (Gassman, 2001). The different characteristics of team members will clearly will affect the ways in which people interact, both cognitively and interpersonally (Kurtzberg \& Amabile, 2001), as well as the ways in which a team will interact. Teams comprising of individuals from different professional backgrounds with different knowledge, skills and abilities, will be more innovative than those whose members have more in common, precisely because they bring differing useful perspectives on issues to their teams (Paulus, 2000).

\subsection{Creativity within GDT}

Analysing the literature dedicated to GDT, there are only a few studies dedicated to the analysis of the creative process of distributed teams, and not much is known about the structures that foster the creativity of New Product Development teams (Leenders, Van Engelen \& Kratzer, 2003).

There are few studies devoted to analysing the creative process in GDT. Nemiro's research (2002), which is amongst the most quoted and referenced, explores how the creative process evolves in distributed teams. Leenders et al. (2003), with a quantitative approach based on a social network perspective, attempted to further understand how creativity in new product development (NPD) teams may be fostered, both in settings of low and high virtuality. Kristensson \& Norlander (2003) conducted an experiment with 96 students in the Cyber/IT Research Laboratory of Karlstad University. Their main aim was to investigate the effects of electronic interaction of groups over individuals in terms of their creative performance. Although a great deal of attention has been paid to various characteristics of GDT, there remains much to be investigated concerning the phenomena inherent in the creative process.

Creativity does not actually occur in the mind, but in the intersection between a person's thoughts and a socio-cultural context (Feldman, Csikszentmihalyi, \& Gardner, 1994). The feasibility of distributed teams was confirmed by Lenders et al. (2003), and this feasibility becomes especially apparent when tackling the incremental tasks of innovation; where the function of a product can be decomposed into semi-independent components corresponding to its many functional parts. Grevins (2002) suggests that the geographical distribution of the team is a significant predictor of project team processes and, consequently, of project performance. The author defines cohesion and conflict as strong components impacting upon the psychosocial outcomes of the team, and addresses the negative effect of conflict in the results of the project. On the other hand, trustiness is perceived by Jarvenpaa \& Leidner (1999) as a paradox because whilst there is the stereotypical view that "trust needs touch" (Handy, 1995), only trust can prevent the geographical and 
organizational distances of global team members from becoming psychological distances.

Some measures are recommended to minimise the effect of the "global" element upon GDT. Oertig \& Buergi (2006) stress the importance of constant dedication to idiom and intercultural communication training: even when sharing the same spoken idiom, the interpretation of signifiers in the implied sense of the words might be understood in quite different forms (Baba et al. 2004). Members with different cultural backgrounds reveal divergent preferences for social interaction norms, which can create difficulties in executing processes related to task integration (Maznevsky \& Chudoba, 2000).

Another important issue to clarify in GDT is the concept of work environment. Some researchers have attempted to further investigate the role of the work environment in the creative process within collocated teams, as well as identifying the elements that influence creativity in a team. Although the work environment is perceived as an ethereal element, it exists regardless of such perception, and is considered to be an attribute of every organisation (Kwasniewska \& Necka, 2004). Such perception exercises a crucial influence upon both the motivation and the creativity of the team (Amabile, 1996).

Since the 1980s, studies dedicated to assess the influence of the work environment upon creativity have grown in strength. According to Kurtzberg and Amabile (2001), individuals produce lower levels of creativity when they perceive their work environment as being constraining or controlling, whilst creativity is enhanced in one that is perceived as being comfortable and psychologically unthreatening (Steiner, 1972).

For this study, the concept of the work environment represents the "organizational climate", which results from a series of elements acting upon the creative performance of the team. It is important to highlight that the elements of which the work environment is composed may or may not directly contribute towards the creative process. Nevertheless, the lack or deficiency of such elements may cause apathy amongst the participants, resulting in creativity losses.

\section{Research Design \& Setting}

The research objective of this study is to explain how the creative process takes place in GDT. Our purpose is to understand the community being researched; we adopted the Case Study method. This method is appropriate for studying contemporary events where the relevant behaviors cannot be manipulated, and wherever it is possible to entail direct observations and systematic interviews (Yin, 1989). Since this study focuses on a globally distributed phenomenon, it was imperative that the selected organisation not only had sites in different countries, but also teams working effectively together in different locations. Consequently we selected the company Volvo 3P in Brazil, which works with teams distributed in Sweden, France and India.

With values of quality, safety and environmental care, Volvo Group is one of the leading suppliers of commercial transport and transport solutions, providing such as trucks, buses, construction equipment, and drive systems for marine and industrial applications as well as aircraft engine components. The Group has about 100,000 employees, production facilities in 20 countries, and sales activities in some 190 markets (Volvo Group Annual Report, 2011). After the acquisition of Renault Truck Corporation on January 2001, and upon a complete review of its structure, Volvo introduced the concept of business areas and units.

The focus of this present research is on "trucks", which in 2011 corresponded to a 64\% share of the total SEK M 310,367 net sales made by the Volvo group. Based upon the concept of cross-functional working groups, the teams allocated for the development of new products for Trucks Volvo (except for PowerTrain) are based at Business Unit Volvo 3P. They work with Volvo Trucks (which is responsible for the manufacturing process), and Volvo PowerTrain (the business unit responsible for developing and manufacturing PowerTrain).

The Business Unit Volvo 3P incorporates Volvo Trucks, Renault Trucks, Mack Trucks, and Nissan Diesel Trucks. A cross-functional organisation, it is responsible for product development, purchasing, product planning and product range management. Volvo emphasizes business and customer focus; speed, agility and flexibility; diversity; teamwork; leaders as coaches; and openness to change. It considers employees as key for the success of the organization. In addition to their excellent technical skills, the collaborators are multi-brand and multi-cultural minded.

Volvo 3P has 4,980 employees distributed across offices based around the world: 1800 in Gothenburg (Sweden), 1160 in Lyon (France), 530 in Greensboro (USA), 210 in Curitiba (Brazil), 640 in Bangalore (India), 50 in Shanghai and 560 in Ageo (China) and 30 in Brisbane (Australia). For the composition of the teams responsible for the development of new products, Volvo has been using both local resources and global resources from various areas. 


\section{Data Collection \& Procedures}

In order to comprehend Volvo's structure, project composition, and the context in which their trucks are developed, we began our research by studying Brazilian pilot project. The outcomes of the first round of data collection indicated the feasibility of studying 15 projects: 10 global projects and 5 local ones, contemplating both product and process. The next step was to restrict the number of projects studied to deepen the research. With this aim in mind we created the selection criteria as follows: a) A focus on the development of new products; b) The nature of the projects themselves (pursuing similar degrees of complexity between collocated and globally); c) Classified as Class III of the Global Development Process - GDP (as defined by Volvo, Class III corresponds to more complex projects); d) Developed in countries with a first language other than English (to put all participants on the same footing with the variable language); e) To have gone through, during the 18 month-period of the interviews, the six stages of the GDP (pre-study, concept study, detailed development, final development, industrialisation \& commercialisation, and follow-up). After applying the criteria set, we wound up with 7 projects to be studied.

Following the theory of building research from a case study (Eisenhardt \& Graebner, 2007; Eisenhardt, 1989), we sought the relationships of occasional circumstances, phenomenon, context, interventional circumstances, interaction actions/strategies, and consequences. The documental analysis enabled the data to be integrated and analysed; which together with direct observation resulted in data triangulation.

\subsection{Selected Projects}

To fulfil the comparative aspect of this research, interviews were conducted with two collocated teams (Sweden and France) and five globally distributed teams (Brazil, Sweden, France, and India) with a total of seven project teams working with chassis and cabins. These projects perform on a cross-functional basis, and therefore have a core structure of members of Volvo 3P and an extended team of representatives from different areas involved in the process as a whole. The extended team comprises of one Chief Project Manager, who is responsible for distributing the load and the project implementation, and one Project Manager (PM) for each designated area involved in the project. This translates as one Development PM, one Manufacturing PM, a Supply PM and so on and so forth. Each PM has the support of a small sub-team of Project Leaders who represent each area. In average each core team comprised of 6 participants, both local and global. However, it is important to pinpoint that this average per team is rather difficult to establish given the complexity, distinct natures and the global organisation of some of these projects. The main goal of this structure is effective project management, whilst the responsibility for execution depends upon each line.

\subsection{Instruments for Data Collection}

The instruments chosen for data collection are the documental analysis, semi-structured interviews and observations.

Archival Data. Job descriptions, human resources' policies, self-managed teams' institutional presentations, internal reports, press coverage and interviews, company's manuals (Code of Conduct, The Volvo Way, Health and Safety policies, Environmental Policies, Volvo Trucks); Institutional presentations of Volvo do Brasil, Volvo Trucks, Volvo 3P, and Volvo PowerTrain; and internal communications such as magazines and other publications. Access was also granted to Volvo's intranet, and to the social accounting and general information available to shareholders on the Internet.

Semi-Structured Interviews. In August 2007, the first contacts with Volvo do Brasil were made via e-mail and phone calls. In November 2007 we visited Volvo's plant in Curitiba-Brazil for the first run of face-to-face interviews. 29 interviews were conducted, each lasting 1 hour 30 minutes on average. In March 2008, contact was restored with the Vice-President of Chassis, Volvo 3P Sweden, who was introduced to my research during a visit to Volvo in Curitiba, Brazil, and who subsequently facilitated visits to Volvo 3P's site in Gothenburg (Sweden) in June 2008, when 18 interviews were held. In November 2008 we visited the Volvo 3P site in Lyon (France), where 15 interviews were conducted. Finally, in December 2008, data collection was completed with three telephone interviews with members of the Volvo 3P from Bangalore (India). In total, we did 65 semi-structured interviews at Volvo do Brasil, Volvo PowerTrain, Volvo Trucks, and Volvo 3P. Of the 65 interviews, 30 were conducted in order to comprehend Volvo's structure, project composition, and the context in which its trucks are developed; and the remaining 35 were conducted for the 7 projects chosen for this research, of which 31 were made with Project Managers (PM's) and Chiefs of Project Managers (CPM's) of the GDT's, and 4 with the PM's of the collocated teams, totalling an average of 5 team-members per project. With the consent of the participants, interviews were recorded and transcribed verbatim.

Observations. During the interviews in Brazil we were invited to observe a project workshop. In this occasion the organizers, through simulation techniques, invited the participants (from Volvo Trucks, Volvo 3P, Concessionaries Volvo and Volvo Powertrain) to recreate the alteration of a "process concept" through simulation techniques. This 
particular event lasted for eight hours was attended by 22 participants, comprising members of the project team and guests from other areas involved with the modifications under discussion. This workshop was held at the Viking Association in Curitiba. In addition, we observed the First Workshop in Innovation \& Technology promoted by VdB, where 11 projects on innovation were presented. This event was held at University Positivo, and lasted for eight hours. Notes were taken during these events to register all the phenomena and processes that were taking place both in the individual and collective spheres (Glaser, 1978). Field notes were transcribed into Word files and then coded. In both workshops we were invited to stay and have lunch and coffee breaks with the participants, which allowed for a more informal and open contact with attendees. From November 19th to 30th, in Brazil, and then from June 17th to 28th, in Sweden, and again between November 24th and 25th and on December 4th in France, we shared lunch with employees at the organization's restaurant, occasions where valuable insights about their work experiences were forthcoming.

\section{Data Analysis}

We have chosen theory building from case study research to analyze the data collected. According to Eisenhardt \& Graebner (2007), it can be applied to one or more empirical cases to create theoretical constructs, propositions and/or midrange theory. The theory is emergent in the sense that it is situated in and developed by recognizing patterns of relationships among constructs within, and across cases and their underlying logical arguments.

In this research we have opted for the 'Content Analysis' technique as a text analysis tool due to the fact that it is via discourse that we create the new ideas, theories and liaisons through which we understand the world and relate to one another. For Krippendorff (2004), the content analyst views data as representations not of physical events but of texts, images, and expressions that are created to be seen, read, interpreted and acted upon for their meanings, and must therefore be analyzed with such uses in mind. Regarding the style of the content analysis, we have opted for a structural approach to confirm the relationship of association among ideas to various macro outcomes (Kennedy, 2008).

Three different categories that help us better perceive how the creative process evolves in GDT have emerged from the analysis we carried out during the codification of the data collected. They are, respectively, the foundations of the creative process (to better analyse this category we have adopted a comparative perspective between GDT and collocated teams), the process of generation of ideas and the interferences and interactions that have been pointed out as significant by interviewees. The second codification was based upon those two above mentioned categories and it was intended to identify the singular contribution of those elements within the global composition of the process. This has allowed a broad overview of the elements that are essential for the creative process.

\subsection{Procedures}

We adopted two stages to analyse the data; firstly we qualify the data and secondly we applied the content analysis.

Stage 1: Qualifying Data. We did two classifications. In the first one our objective was to see the phenomena "between the lines" in order to understand the intricacies of the company and the context. From this classification we start to build some concepts about the design of the analysis.

In the first classification we divided the documents per company and project. By company (Volvo do Brasil; Volvo Powertrain; Volvo Truck - Brazil; Volvo 3P - Brazil; Volvo 3P - Sweden; Volvo 3P - France; Volvo 3P - India). And by classification of project (Product and Service).

In the second classification, our aim was to compare the development of new product firstly in terms of the process and secondly in terms of the countries. With this classification it was possible to identify the subtleties of cultural differences in the creative process. In cases where the interviewee was an integral member of more than one project, the interview was divided. In this way each project had its commentary separately.

Stage 2: Content Analysis. The data collected was submitted to a lexical analysis in order to verify the word frequency and connections. Our objective was to identify the most cited constructs. We use TALTAC software: it is software for automatic analysis of text in the dual logic of Text Analysis (TA) and Text Mining (TM).

As a result of lexical analysis, the Table 1 shows the 50 most cited words, grouped in accordance with the sets associated with people (whose emphasis are as much on the individual as on the team (we inserted the word 'together' here in the sense of 'joint action'); words correlated to the organisation; task (projects for developing new products); regarding to internal process; communication; words associated directly to the word 'problem' (without emission of judgment); words correlated to the solution; and finally we created the set 'various' were we added the words that, for a more accurate inclusion, an analysis of context was necessary.

From this classification we can look to identify the context which they are inserted. We used as search criteria an 
interval of five words preceding and following the word in question. The results can be found in the Table 2 .

Table 1. List of 50 of the Most Cited Words in Sets

\begin{tabular}{lclccccc}
\hline \multicolumn{1}{c}{ INDIVIDUAL } & Count & ORGANIZATION & count & TASK & count & $\begin{array}{c}\text { INTERNAL } \\
\text { PROCESS }\end{array}$ & count \\
\hline Person [people] & 1479 & global[ly] & 365 & project[s] & 1593 & creat[ivity] & 985 \\
team[s] & 760 & Organization & 179 & truck & 420 & Trust & 143 \\
Together & 177 & Cost & 72 & develop[ment] & 402 & Quality & 133 \\
Manager & 163 & Countries & 59 & product & 341 & Values & 102 \\
colleagues & 58 & Client & 51 & concept & 176 & Culture & 93 \\
& & & & engin[eering] & 172 & change & 79 \\
& & & & supplier[s] & 147 & knowledge & 60 \\
& & & & GDP & 147 & relationship & 52 \\
\hline COMMUNICATION & Count & PROBLEMS & count & SOLUTIONS & count & VARIOUS & count \\
\hline meeting[s] & 771 & problem[s] & 417 & idea[s] & 227 & time[s] & 583 \\
Language & 118 & differen[ce] & 215 & solution & 192 & Way & 243 \\
information & 91 & limitation[s] & 117 & & & local & 171 \\
communication & 52 & Question & 80 & & & important & 96 \\
& & Discuss & 76 & & & experience & 82 \\
\hline
\end{tabular}

Source: the author.

Table 2. Context of the Most Cited Words by Set

\begin{tabular}{|c|c|c|c|c|c|}
\hline \multicolumn{6}{|c|}{ INDIVIDUAL } \\
\hline person [people] & tecnologies & commitment & Respect & responsability & Information \\
\hline team[s] & Diversity & leader & Cooperation & work & Capabilities \\
\hline together & Building & links & Supporting & foundation & Suppliers \\
\hline manager[s] & employees & guide & Principles & responsability & Improvement \\
\hline colleagues & Efficient & manager & Team & area & Project \\
\hline \multicolumn{6}{|c|}{ ORGANIZATION } \\
\hline global[ly] & Culture & company & Transport & profitable & Growth \\
\hline organization & individuals & values & Brands & energy & Willingness \\
\hline Cost & reduction & effectiveness & competitiveness & project & Truck \\
\hline countries & sales activities & employees & Worldwide & knowledge & emissions standarts \\
\hline Client & Broader & heavy & segment & truck & Brand \\
\hline \multicolumn{6}{|c|}{ TASK } \\
\hline project[s] & Changes & products & development & integrating & distributing resources \\
\hline Truck & environmental & production & launched & engines & Architecture \\
\hline develop[ment] & information & efficiently & competence & product & Sinergies \\
\hline product & $\begin{array}{l}\text { technology } \\
\text { quality }\end{array}$ & safety & environmental care & customers & Vehicles \\
\hline \multicolumn{6}{|c|}{ INTERNAL PROCESS } \\
\hline creat[ivity] & promote & employees & energizing & diversity & Innovation \\
\hline Trust & knowledge & values & relations & communications & Motivation \\
\hline quality & safety & $\begin{array}{l}\text { environmental } \\
\text { care }\end{array}$ & services & values & Goals \\
\hline values & corporate & Volvo way & principles & credibility & Quality \\
\hline culture & best-practice & corporate & factor & importance & Values \\
\hline \multicolumn{6}{|c|}{ COMMUNICATION } \\
\hline meetings & representatives & committees & employe & shareholders & Regular \\
\hline language & communication & business & personal & IT & Problem \\
\hline information & communication & market & financial & value & Knowledge \\
\hline communication & shareholders & stakeholder & supply & follow-up & $\begin{array}{l}\text { new product } \\
\text { developed }\end{array}$ \\
\hline
\end{tabular}




\begin{tabular}{|c|c|c|c|c|c|}
\hline \multicolumn{6}{|c|}{ PROBLEMS } \\
\hline problem[s] & quality & major & technical & $\begin{array}{l}\text { distributing } \\
\text { resources }\end{array}$ & Language \\
\hline differen[ce] & translations & instruments & between & time & Frame \\
\hline limitation[s] & work global & language & time & coordination & Resources \\
\hline question & additional & understood & risk management & think & Way \\
\hline discuss & french & future & investment & strategy & Round \\
\hline \multicolumn{6}{|c|}{ SOLUTIONS } \\
\hline solution[s] & technical & robust & results & creative & Portrait \\
\hline ideas & New concepts & thoughts & exchange & creative & Together \\
\hline
\end{tabular}

Source: the author.

\section{Findings}

In order to explain how the creative process takes place, we present our findings classified in three main categories, the foundations of the creative process in GDT, the process of generation of ideas and the interferences and interactions that have occurred during the creative process.

\subsection{The Foundations of the Creative Process}

The analysis of the teams taking part in this research suggests that communication is the central basis for the creative process in both globally distributed and collocated teams. However, in collocated teams the individual underpins the process, as the resources required for the development of the creative process depend solely on the individuals. On the contrary, in GDT the organization from the basis of the process because it provides the technological resources required to facilitate communication between team members at each site. As a consequence, the organization plays a fundamental role for the creative process of GDTs.

With regard to GDT, two other relevant elements in the foundation of creative process which concern organization are clear values and information technology. Clear values are represented by "Volvo-Way”. The Volvo-Way's manual synthesizes the company's philosophy, values, and corporate culture. A dynamic and long-term conception has been evaluated and improved by encouraging the participation of all employees. During the interviews, some Volvo-way mottos were quoted or paraphrased by employees: “... we work with energy, passion, and respect for all the people ...”; "We use our knowledge to develop transport solutions ... with superior levels of quality, safety, and respect for the natural environment ...”; “... we act focusing the client with open dialogue, the spirit of the enterprise and team work ...”. In addition, we have noticed that the Group's principles are continually reinforced through several management mechanisms, including Quality Control Policies, Environmental Policies, Working Environment Policies and Volvo do Brasil's code of conduct.

In relation to information technology, we have found forums and councils responsible for evaluating new technologies and demands on a systematic and permanent basis. According to all our interviewees there are two aspects that either directly or indirectly facilitate working in globally environments: access our interviewees there are two aspects that either directly or indirectly facilitate working in globally environments: access to information and access to tools of communication. With regard to employees' access to information, in Brazil information is disseminated and made available on a broad basis and with constant improvements, interlinking all areas through: electronic media; the Viking Mural; the Corporative TV; and the Volvo Magazines. There is also the system of Archives, which is responsible for storing all documentation and keeping a directory of all publications, such as books, journals, and norms.

With respect to the access to tools of communication used by GDT members, we have found phone systems, NetMeetings, video conferences, and MSN. The obstacles faced in the course of using these means of communication, such events as system failure or even policies to rationalize costs, are considered rare:

"Problems with system failure? It very rarely happens. A satellite needs to fail or there is a problem with cabling. Here in Brazil we have submarine communications cable. Really, it is not an issue. In seven years working for Volvo, I think it happened twice or maybe three times at a push”. (R.B., personal communication, November 2007).

One aspect emphasized was Volvo's commitment in constantly updating and providing new technologies to facilitate cross-country communication, such as Live Meeting, a web conference service operated by Microsoft:

"Up to now, NetMeeting has presented some problems, for example: you have the screen up and running, you are 
using CAD, and you want to show something to your colleague. You should be able to rotate images, but you cannot do that. Now, Live Meeting is software that allows you to do that, it is like a second generation of NetMeeting. For example, I will be able to work in CAD and in real time; someone in France can see what I am doing. "Look, now I am applying the radius here." "Done” ... the other person in France can see what I have just done and say if it is good enough or not. He can, for example, take control and access the same database. It is cross-ocean interaction... very soon we will not be able to justify business trips any longer. Technological interaction is now immense”.(M.S., personal communication, November 2007).

Through both the transparency of their objectives and their cutting-edge information and technology systems, we may therefore affirm that the organization successfully provides their GDT with tools and environments that foster the development of the creative process. High levels of motivation amongst individuals responsible for developing new products immediately become clear. Their interest goes beyond the limits of their own project and extends to a broader view of what is being developed by other teams, right down to who is taking part and what their objectives and targets might be. This attribute was identified in all interviewees. There was a spark in their eyes when they described, in rich detail, their activities and responsibilities; commitment to their projects and personal satisfaction taken from the creative process were obvious. An intrinsic motivation to work in a distributed environment was also evident when interviewees espoused the benefits of the technological advances provided by this type of environment. In this context, the individual gives space to the team. According to one of Powertrain's coordinators, "Nothing is individual. You have to make a few small decisions in order to keep the process moving, but decisions are always made by the team."

Both the organization and the individual contribute towards the development of a harmonious and balanced team. Summarizing this point, the creative process in teams is grounded in "communication". In collocated teams the individual is the axe, while in GDT the axe is the organization. We can assume that clear project goals, shared values, and vision commitment are variables that affect communication in both teams, collocated and globally distributed. Different workplaces have an impact on communication, technology and intrinsic motivation, specifically in GDTs. These differences are associated with the environments of GDTs, which demand a greater commitment from the participants.

Generation of ideas. We analysed the interviewees' answers about the activities developed throughout the execution of the projects, as well as how and when the ideas are generated within the team. From our findings we can verify that the process of generation of ideas process within a team is identified as a 'looping process' where research and debates are combined, leading the participants to pursue either an individual or a shared creative process. In the end, the idea itself does not involve exclusive ownership as it results from the participants' interactions. Yet we noticed yet that the element 'time' is required to allow those ideas to blossom. Very often the gestalt is opened during meetings but may only be closed outside the work environment:

"I suppose that most of the creative work being done in the development team is not being done in our meetings, but in between the meetings.” (F.S., personal communication, June 2008).

"It's difficult to explain it, how the new ideas come out. Coincidently, I think it's completely impossible. You ever think that most of people who have new ideas have these ideas when they are not working in the office? Cannot even try thinking of it when they are working...: But walking and pondering or walking in your garden, or whatever? Or whatever... But even through the woods and so on. That's you, you never know, how the idea comes, but I think... Because, I think an idea is the meeting point of different visions. (S.F., personal communication, November 2008).

In collocated teams, an informal exchange of ideas takes place in corridors and in the staff kitchen; they arise from informal chats occasioned by the physical proximity of the working environment. Consequently, ideas can be processed in-group, even if it has a self-processing character. For GDT, the communication process is assisted by technology; therefore the intrinsic informality of exchanging ideas assumes a formal character. Even when the tone is kept informal, one must have the initiative of picking up the phone or writing an e-mail. With a lack of face-to-face interaction, there is more room for misunderstandings, and although the loop remains the same it tends to be more elaborate for GDT. There is no casualness, and more commitments are required from the team-members. At this stage, "intrinsic motivation" appears crucial if one wishes to take part in GDT, since it demands a differential attitude from its members and more individual commitment.

"I think that we see that we have the same target, so that we are all out trying to think that we understand our different situations. What is hard in the factory side, what is rather hard in PD side? So, we understand a little bit about others' jobs that and we try to help them. (C.R., personal communication, June 2008). 
Figure 1 shows the loop for the generation of ideas in teams. The essential difference between projects developed by GDT and projects developed by collocated teams is the "informality" of the environment for exchanging ideas, and the casualness of the process. The question mark represents the starting point of the project to be developed. Upon achieving the final result (that is the alteration or creation of a new product), we investigate the trajectory of the team towards the final goal. The 1st step represents the research carried out individually by team members on similar projects. Upon completion of this stage all information gathered goes through a 'maturing' process, i.e., the information gathered is conciliated and new ideas are produced. Then, in an informal manner, the team-members exchange ideas and experiences: this interaction results in new concepts that will be assimilated on an individual basis. Following this, the team takes part in a formal meeting where conclusions and new concepts are shared. After this meeting, team-members reprocess the outcomes of the formal meeting and a new research on the implications is automatically triggered. The looping therefore recommences and it will only end when a 'new idea' becomes an applicable concept.

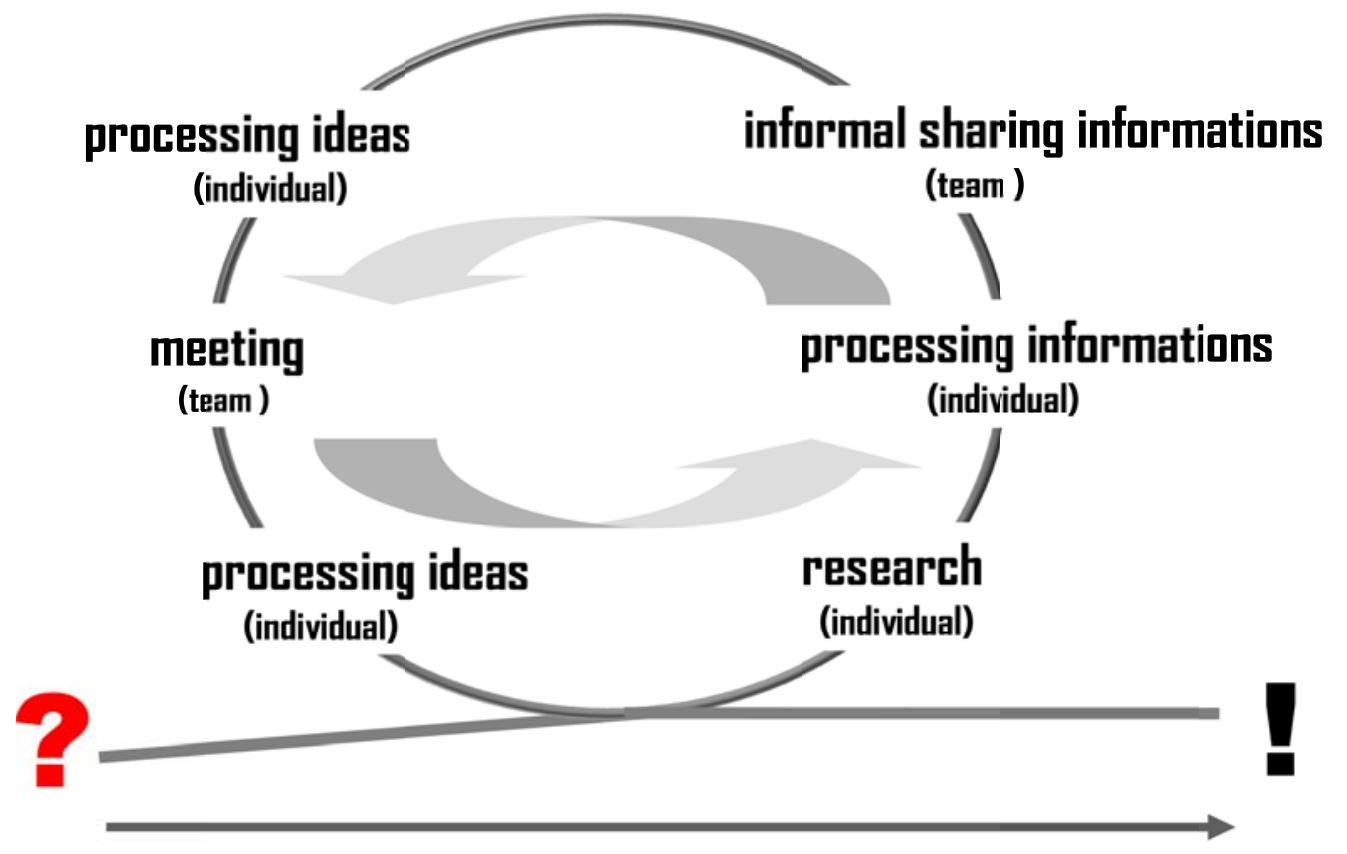

Figure 1. The Looping Process in Generating Ideas

Source: the author.

\subsection{Interferences and Interactions}

Language was identified as the most difficult aspect. Most interviewees mentioned the frustration that using a second language can involve. They pinpointed differences in English proficiency amongst team members, and how the lack of body language more often than not constrains communication. As the grasp of English is not uniform, misunderstandings leading to frustration become common place. "You can use the same word and understand them differently..." To solve this problem some organizational tools were introduced, such as taking minutes of every conference call. Similarly, exchanging e-mails and reports is a schematic form that has strengthened trust among participants: written communication had been introduced to reinforce and give support to verbal communication.

The time zone factor is regarded as both an advantage and a disadvantage. Appearing as a problem if prompt feedback is required, it can nevertheless facilitate the creative process when team members process information at their own pace. The interviewees stressed the difficulties of holding a conference call with all members after Japan joined the team, concluding that "If you wish to work in GDT, you'll find the time".

Cultural diversity in certain types of team compositions can either trigger conflicts and/or promote collaboration, which, either way, enhances and benefits the creative process. The collaboration amongst team members is seen as crucial for the actual market. Taking into account the multiple geographic units that Volvo holds worldwide, diversity is perceived as being positive to integration, and a fruitful source of knowledge from which its workforce can learn. 
We have different cultures that we need to learn from each other. If I say something, you will probably interpret that in your way, with your background and maybe that wasn't what I was meaning and we need to learn these things from each other. The more we work together, the more we learn from each other and learn how we are thinking and it's the way to move forward, I mean. (J.S., personal communication, December 2008).

The diverse background of team members in GDTs allows for a fruitful integration of knowledge, whilst time zone differences and formalization procedures slow down the process. As restraining factors that affect the creative process, participants identified "time" and "knowledge market" as inhibitors of the creative process in GDTs and collocated teams respectively.

\section{Discussion}

We stressed here some points that we consider to be either complementary or inconsistent within the existing research. In the late 1990s some researchers devoted a great deal of effort to pinpointing the restrictive and constraining aspects of the creative process in GDT, although today some of these aspects are seen as nothing but manageable noises in the communication process.

Various challenges faced by members of GDT such as the use of a second language use, time zones and cultural diversity become part of the daily life of the organization. Each of these hurdles has enlightened and enriched scholarly debate, promoting different perceptions and approaches. Although such aspects are commonly identified as obstacles for GDT, nowadays they are all manageable. Moreover, it has been shown that cultural diversity has been contributed to efficiency and success.

One ought to take into account the fact that the technological tools of communication are subject to continual and rapid advancements. For example, video conference tools considered as cutting-edge technology as recently as 2002 are now regarded as a rather obsolete resource, and slowly but surely are being largely replaced by Microsoft "live-meeting”: a web conferencing service that enables users to collaborate and share in real time with only a PC and Internet connection. Therefore, some of the points regarded above as being obstacles to communication and factors for conflict may soon become largely or even entirely irrelevant.

According to Chen (2006), creativity in project teams is a complex social system since it involves a combination and integration of various inputs and knowledge from multiple and interdependent project team members. Although Chen's study concerned only collocated teams, the findings demonstrated from the present research show that this affirmation can be extended to GDT.

In the absence of specific explanatory models applicable to the creative process in GDT, we have sought support in the literature dedicated to collocated teams for insights on how better to understand the phenomenon in question, and for this we adopted a componential approach. Amabile's $(1983,1996)$ componential model of creativity predicts that three components contribute to creativity: task motivation, domain-relevant skills and creativity-relevant processes. For Sternberg \& Lubart (1999), creativity requires a confluence of six distinct but inter-related resources: intelligence, knowledge, cognitive styles, personality, motivation and environmental context. Lubart (1999) also mentions two more relevant approaches that could be considered as componential: the Interactionist Model developed by Woodman \& Schoenfeldt (1989, 1993) and the Systems Approach of Feldman, Csikszentmihalyi \& Gardner (1994) and Csikszentmihaly (1996). In the first model, creative behavior results from a complex interaction between a person and a situation. This model is composed of three main components: antecedent conditions, the characteristics of the person, and the characteristics of the situation. For the Systems Approach of Feldman et al. (1994), the individual (first system) draws on information in a domain and transforms or extends it via cognitive processes, personality traits and motivation. The field (second system), consists of people who control or influence a domain, and evaluate and select new ideas. The domain (third system), consists of a culturally defined body of knowledge which incorporates creative products and can be transmitted from one person to another.

Following the componential approach, an argument to be discussed would be the identification of the basic components of the tripod supporting the creative process in GDTs. In the literature dedicated to collocated teams, we have found consensus in only two axioms: the individual (Woodman et al., 1989-1993; Feldman et al., 1994), and the domain (Amabile, 1983- 1996; Feldman et al., 1994, Csikszentmihaly, 1996). Other elements such as the team, the organization, skills, and the field have also been listed. Comparatively, in our research, we have implied that technology, tasks, and motivation are the basic components for the creative process in GDT. 


\section{Conclusion}

The present research is built upon the case study of an organizational setting, based upon which we attempt to grasp how the creative process occurs and how it can be explained within GDT engaged in the development of new products. Regarding the core of the creative process, we have identified the axiomatic role of communication, the weight of the organization and some unique aspects of the creative process in GDT. Most noticeable on the individual level is the role played by motivation and the members' predisposition to work in GDT. On a team level we have identified facilitating aspects such as shared objectives, norms and values, and clear project goals.

Our exploratory research suggests that it is information technology alongside with intrinsic motivation and task which lay down the necessary foundations for the development of the creative process in GDT. During our research we came to the realization that the evolution of technology has immensely improved the quality of communication between team members. Intrinsic motivations, the willingness to work in a globally distributed workplace, plus the creative process in GDT represent a mix between individual and team work. Modular tasks represents the amalgam that keeps members of the project together; modular activities allow development in GDTs, for it provides a plan for the project that has clear and monitored objectives.

Factors such as language, time-zones, and cultural differences do not appear to create obstacles, but instead allow participants in GDT to take advantage of the positive aspects of these factors. For instance cultural diversity allows a greater diversification not only of knowledge itself but of its application, and time-zones allow participants to take advantages of calendar differences, such as different holidays for South America and Europe. These factors keep the project away from inertia.

In addition, it is possible to assert that the quality of interpersonal relations is affected by how clearly the objectives are explained and conveyed to the team, and by how the participants understand their roles in the process. This therefore facilitates the development of trust between participants, which is of paramount importance to the creative process.

\section{Limitations and Avenues for Future Research}

Some limitations warrant a mention before avenues for future research are discussed. Firstly, with regard to data collection, this type of research does not allow room for ubiquities, since GDT members are geographically distributed during the creative process. In this sense, observations can only be made from a unilateral perspective.

As this research is based upon a qualitative approach, generalization is rather restricted. Results cannot be extended with the same certainty to a broader population. If the results of the present research are therefore to be applied to the study of other problems in different fields, it would be essential to identify some grouping categories. With a single case as a starting point, one should clarify all circumstances and situations, submitting them to comparison exercises, in order to pursue an "argued generalization".

Future researchers are also encouraged to address the comparison between product and process. New investigations are needed to unravel the complexity of the creative process in 'new processes'. The absence of a product or the lack of concreteness of some elements that can be touched and felt with 'one's hands' when one is engaged in an abstract discussion along with the distance imposed upon team members, represent an argument yet to be explored.

\section{References}

Amabile, T.M. (1983). The social psychology of creativity. New York: Springer-Verlag NewYork Incorporated. http://dx.doi.org/10.1007/978-1-4612-5533-8

Amabile, T.M. (1996). Creativity in context. Boulder: Westview Press.

Amabile, T. M., \& Amabile, T. M. S. P. O. C. (1996). Creativity in context: update to The social psychology of creativity. Boulder, Colo; Oxford: Westview Press.

Baba, M.L., Gluesing, J., Ratner, H., \& Wagner, K. (2004). The contexts of knowing: Natural history of a globally distributed team. Journal of Organizational Behavior, 25, 547-587. http://dx.doi.org/10.1002/job.259

Chen, M. (2006). Understanding the benefits and detriments of conflict on team creativity process. Creativity and Innovation Management, 15(1), 105-116. http://dx.doi.org/10.1111/j.1467-8691.2006.00373.x

Cini, M.A. (1999). Learning leadership online: A synergy of the medium and the message. The Journal of Leadership Studies, 5(2), 103-115. http://dx.doi.org/10.1177/107179199900500209 
Csikszentmihalyi, M. (1996). Implications of a systems perspective. In Sternberg RJ (ed.) Handbook of Creativity. Cambridge: Cambridge University Press.

Eisenhardt, K.M. (1989). Building theories from case study research. The Academy of Management Review, 14(4), 532-550.

Eisenhardt, K.M., \& Graebner, M.E. (2007). Theory building from cases: Opportunities and challenges. The Academy of Management Journal, 50(1), 25-32. http://dx.doi.org/10.5465/AMJ.2007.24160888

Feldman, D. H., Csikszentmihalyi, M., \& Gardner, H. (1994). Changing the world: a framework for the study of creativity. Westport, Conn; London: Praeger.

Gassman, O. (2001). Multicultural teams: Increasing creativity and innovation by diversity. Creativity and Innovation Management, 10(2), 88-95. http://dx.doi.org/10.1111/1467-8691.00206

Glaser, B.G. (1978). Theoretical sensitivity: Advances in the methodology of grounded theory. Sociology Press.

Grevins, J. (2002). Project virtuality effects on project team process and project success. Unpublished doctoral dissertation, University of New York, Buffalo.

Handy, C. (1995). Trust and the virtual organization. Harvard Business Review, 3, 40-50.

Jarvenpaa, S.L., \& Leidner, D.E. (1999). Communication and trust in global virtual teams. Organization Science, 10(6), 791-815. http://dx.doi.org/10.1287/orsc.10.6.791

Kennedy, M.T. (2008). Getting counted: Markets, media, and reality. American Sociological Review, 73, 1021-1021. http://dx.doi.org/10.1177/000312240807300609

Krippendorff, K. (2004). Content Analysis - an introduction to its methodology. Thousand Oaks: Sage Publications Inc.

Kristensson, P., \& Norlander, T. (2003). The creative product and the creative processes in virtual environments. Creativity and Innovation Management, 12(1), 32-40. http://dx.doi.org/10.1111/1467-8691.00266

Kurtzberg, T.R., \& Amabile, T.M. (2001). From Guilford to creative synergy: Opening the black box of team-level creativity. Creativity Research Journal, 13(3-4), 285-294. http://dx.doi.org/10.1207/S15326934CRJ1334_06

Kwasniewska, J., \& Necka, E. (2004). Perception of the climate for creativity in the workplace: The role of the level in the organization and gender. Creativity and Innovation Management, 13(2), 187-196. http://dx.doi.org/10.1111/j.0963-1690.2004.00308.x

Kylen, S., \& Shani, A.B. (2002). Triggering creativity in teams: An exploratory investigation. Creativity and Innovation Management, 11(11), 17-30. http://dx.doi.org/10.1111/1467-8691.00233

Leenders, R., Van Engelen, J.M.L., \& Kratzer, J. (2003). Virtuality, communication, and new product team creativity: A social network perspective. Journal of Engineering and Technology Management, 20, 69-92. http://dx.doi.org/10.1016/S0923-4748(03)00005-5

Lubart, T.I. (1999). Creativity across cultures. In Stenberg RJ (ed.), Handbook of Creativity. Cambridge: Cambridge University Press.

Maznevsky, M.L., \& Chudoba, K.M. (2000). Bridging space over time: Global virtual team dynamics and effectiveness. Organization Science, 11(5), 473-492. http://dx.doi.org/10.1287/orsc.11.5.473.15200

Nemiro, J. (2002). The creative process in virtual teams. Creativity Research Journal, 14(1), 69-83. http://dx.doi.org/10.1207/S15326934CRJ1401_6

Oertig, M., \& Buergi, T. (2006). The challenges of managing cross-cultural virtual project teams. Team Performance Management, 12(1/2), 23-30. http://dx.doi.org/10.1108/13527590610652774

Paulus, P.B. (2000). Groups, teams and creativity: The creative potential of idea-generating groups. Applied psychology: An International Review, 20(2), 237-262. http://dx.doi.org/10.1111/1464-0597.00013

Peelle III, H.E. (2006). Appreciative inquiry and creative problem solving in cross-functional teams. The Journal of Applied Behavioral Science, 42, 447-467. http://dx.doi.org/10.1177/0021886306292479

Piccoli, G., Powell, A., \& Ives, B. (2004). Virtual teams: Team control structure, work processes, and team effectiveness. Information Technology \& People, 17(4), 359-379. http://dx.doi.org/10.1108/09593840410570258 
Plucker, J.A., \& Renzulli, J.S. (1999). Psychometric approaches to the study of creativity. In Sternberg RJ (ed.), Handbook of Creativity. Cambridge: Cambridge University Press.

Steiner, I. (1972). Group process and productivity. New York: Academic.

Sternberg, R.J., \& Lubart, T.I. (1999). The concept of creativity: prospects and paradigms. In Stenberg RJ (ed) Handbook of Creativity. Cambridge: Cambridge University Press.

Woodman, R.W., Schoenfeldt, J.E., \& Griffin, R.W. (1993). Toward a theory of organizational creativity. The Academy of Management Review, 18(2), 293-321.

Woodman, R.W., \& Schoenfeldt, J. E. (1989). Toward a theory of organizational creativity. The Academy of Management Review, 18(2), 293-321.

Yin, R. (1994). Case study research: Design and methods. Beverly Hills, CA: Sage. Publishing. 